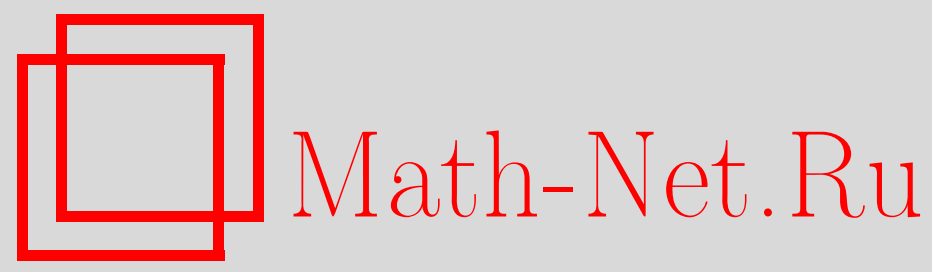

А. Н. Чупрунов, И. Фазекаш, Аналог обобщенной схемы размещения. Предельные теоремы для максимального объема ячейки, Дискрет. матем., 2012, том 24, выпуск 3, 122 129

DOI: https://doi.org/10.4213/dm1203

Использование Общероссийского математического портала Math-Net.Ru подразумевает, что вы прочитали и согласны с пользовательским соглашением http://www.mathnet.ru/rus/agreement

Параметры загрузки:

IP: 52.90 .164 .192

26 апреля 2023 г., 12:02:05 
УДК 519.2

\title{
Аналог обобщенной схемы размещения. Предельные теоремы для максимального объема ячейки
}

() 2012 г. А. Н. Чупрунов, И. Фазекаш

\begin{abstract}
Рассматривается аналог обобщенной схемы размещения, в котором вместо условия, что количество частиц равно $n$, используется условие, что количество частиц не превосходит $n$. Получены предельные теоремы для максимального числа частиц в ячейке.
\end{abstract}

\section{1. Введение}

Пусть $\xi_{0}, \xi_{1}, \xi_{2}, \ldots, \xi_{N}-$ независимые одинаково распределенные невырожденные случайные величины, принимающие значения в множестве неотрицательных целых чисел $\mathbf{N}_{0}$. Мы будем предполагать, что случайная величина $\xi_{0}$ имеет распределение

$$
p_{k}=\mathbf{P}\left\{\xi_{0}=k\right\}, \quad k \in \mathbf{N}_{0},
$$

и

$$
\mathbf{E} \xi_{0}=a<\infty
$$

Напомним, что случайные величины $\eta_{1}^{\prime}, \ldots, \eta_{N}^{\prime}$, имеющие совместное распределение

$$
\mathbf{P}\left\{\eta_{1}^{\prime}=k_{1}, \ldots, \eta_{N}^{\prime}=k_{N}\right\}=\mathbf{P}\left\{\xi_{1}=k_{1}, \ldots, \xi_{N}=k_{N} \mid \sum_{i=1}^{N} \xi_{i}=n\right\}
$$

образуют обобщенную схему размещения. Понятие обобщенной схемы размещения было введено В. Ф. Колчиным в [1]. Многие схемы комбинаторной теории вероятностей (схема размещения различимых частиц по ячейкам, схема размещения неразличимых частиц по ячейкам, случайные перестановки, случайные отображения, случайные графы, и т. д.) являются частными случаями обобщенной схемы размещения [2]. Изучению обобщенной схемы размещения посвящено большое количество работ (см. [2, 3] и их библиографии).

Мы будем рассматривать случайные величины $\eta_{1}, \ldots, \eta_{N}$, имеющие совместное распределение

$$
\mathbf{P}\left\{\eta_{1}^{\prime}=k_{1}, \ldots, \eta_{N}^{\prime}=k_{N}\right\}=\mathbf{P}\left\{\xi_{1}=k_{1}, \ldots, \xi_{N}=k_{N} \mid \sum_{i=1}^{N} \xi_{i} \leqslant n\right\} .
$$


Обобщенная схема размещения интерпретируется как схема размещения $n$ частиц по $N$ ячейкам. Поэтому схему (3) можно интерпретировать как обобщенную схему размещения не более чем $n$ частиц по $N$ ячейкам.

Классическими задачами комбинаторной теории вероятностей являются изучение случайной величины - числа ячеек, содержащих $r$ частиц и случайной величины - максимального числа частиц в ячейке. Для схемы (3) число ячеек, содержащих $r$ частиц, есть случайная величина

$$
\mu_{n N}^{(r)}=\sum_{i=1}^{N} \mathbf{I}_{\left\{\eta_{i}=r\right\}},
$$

где $\mathbf{I}_{\left\{\eta_{i}=r\right\}}$ - индикатор события, состоящего в том, что случайная величина $\eta_{i}$, определенная в (3) равна $r$. Здесь $r-$ фиксированное целое неотрицательное число. Максимальное число частиц в ячейке в схеме (3) есть случайная величина

$$
\eta_{(n N)}=\max _{1 \leqslant i \leqslant N} \eta_{i}
$$

В [4] получены законы больших чисел и локальные предельные теоремы для числа ячеек, содержащих $r$ частиц в схеме (3) - случайных величин $\mu_{n N}^{(r)}$. Настоящая работа примыкает к [4]; здесь мы получим предельные теоремы для случайных величин $\eta_{(n N)}$; в доказательствах теорем настоящей работы существенно используются леммы из [4].

Пусть

$$
\eta_{(n N)}^{\prime}=\max _{1 \leqslant i \leqslant N} \eta_{i}^{\prime}
$$

- максимальный объем ячейки в обобщенной схеме размещения. Пусть $\xi_{0}^{(\leqslant r)}-$ случайная величина с распределением

$$
\mathbf{P}\left\{\xi_{0}^{(\leqslant r)}=k\right\}=\mathbf{P}\left\{\xi_{0}=k \mid \xi_{0} \leqslant r\right\},
$$

и пусть $\xi_{i}^{(\leqslant r)}, i=1, \ldots, N,-$ независимые копии $\xi_{0}^{(\leqslant r)}$. Положим

$$
S_{N}^{(\leqslant r)}=\sum_{i=1}^{N} \xi_{i}^{(\leqslant r)}, \quad a_{(\leqslant r)}=\mathbf{E} \xi_{0}^{(\leqslant r)} .
$$

Для обобщенной схемы размещения (см. лемму 1.2.2 в [2]) справедливо равенство

$$
\mathbf{P}\left\{\eta_{(n N)}^{\prime} \leqslant r\right\}=\left(1-P_{r}\right)^{N} \frac{\mathbf{P}\left\{S_{N}^{(\leqslant r)}=n\right\}}{\mathbf{P}\left\{S_{N}=n\right\}}
$$

для всех $r \in \mathbf{N}$, где

$$
P_{r}=\mathbf{P}\left\{\xi_{0}>r\right\}
$$

Мы будем использовать следующий аналог этой формулы для схемы (3).

Теорема 1. Справедливы равенства

$$
\begin{aligned}
& \mathbf{P}\left\{\eta_{(n N)} \leqslant r\right\}=\left(1-P_{r}\right)^{N} \frac{\mathbf{P}\left\{S_{N}^{(\leqslant r)} \leqslant n\right\}}{\mathbf{P}\left\{S_{N} \leqslant n\right\}} \quad r \in \mathbf{N}, \\
& \mathbf{P}\left\{\eta_{(N)} \leqslant 0\right\}=\frac{\left(1-P_{0}\right)^{N}}{\mathbf{P}\left\{S_{N} \leqslant n\right\}} .
\end{aligned}
$$


Доказательство теоремы 1 повторяет доказательство леммы 1.2.2 из [2]. Теорема 1 позволяет использовать предельные теоремы для сумм независимых случайных величин при доказательстве предельных теорем для $\mathbf{P}\left\{\eta_{(n N)} \leqslant r\right\}$.

Пусть $b_{0}, b_{1}, b_{2}, \ldots$ - такая последовательность неотрицательных чисел, что $R-$ радиус сходимости ряда

$$
B(\theta)=\sum_{k=0}^{\infty} \frac{b_{k} \theta^{k}}{k !}
$$

- положителен. Случайная величина $\xi_{0}=\xi_{0}(\theta)$ имеет распределение

$$
p_{k}=p_{k}(\theta)=\mathbf{P}\left\{\xi_{0}(\theta)=k\right\}=\frac{b_{k} \theta^{k}}{k ! B(\theta)}, \quad k=0,1,2, \ldots
$$

Мы будем предполагать, что случайная величина $\xi_{0}(\theta)$ удовлетворяет условию

$$
b_{0}>0, \quad b_{1}>0 .
$$

Свойство $A_{1}$ и случайная величина $\xi_{0}(\theta)$ были введены в [5]. В [5-7] были получены предельные теоремы для сумм случайных величин $\xi_{i}(\theta)$. Отметим, что

$$
\left.\tilde{m}(\theta)=\mathbf{E} \xi_{0}(\theta)=\frac{\theta B^{\prime}(\theta)}{B(\theta}\right), \quad 0<\theta<R .
$$

В [8] показано, что функция $\tilde{m}(\theta), 0<\theta<R$, обратима.

Теоремы 5-7 являются аналогами теоремы Реньи-Эрдеша [9]. Эти результаты получены для случайных величин $\xi_{i}=\xi_{i}(\theta)$.

\section{2. Основные результаты}

Используя закон больших чисел для оценки числителя и знаменателя дроби в (5), получаем следующую теорему.

Теорема 2. (1) Пусть

$$
d>\max \left(a, a_{\leqslant r}\right) .
$$

Тогда для всех $r \in \mathbf{N}$ при $n, N \rightarrow \infty$

$$
\mathbf{P}\left\{\eta_{(n N)} \leqslant r\right\}=\left(1-P_{r}\right)^{N}(1+o(1))
$$

равномерно по $\alpha_{n N}>d$.

(2) Предположим, что

$$
a<d<d_{1}<a_{\leqslant r} .
$$

Тогда для всех $r \in \mathbf{N}$ при $n, N \rightarrow \infty$

$$
\mathbf{P}\left\{\eta_{(n N)} \leqslant r\right\}=\left(1-P_{r}\right)^{N} o(1)
$$

равномерно при $d_{1}>\alpha_{n N}>d$. 
Используя центральную предельную теорему для оценки числителя и знаменателя дроби в (5), получаем следующую теорему.

Теорема 3. Предположим, что $\mathbf{E} \xi_{0}^{2}<\infty$, и пусть $\sigma^{2}, \sigma_{\leqslant r}^{2}-$ дисперсии случайных величин $\xi_{0}, \xi_{0}^{(\leqslant r)}$ соответственно. Пусть $-\infty<C \leqslant \infty$. Тогда для всех $r \in \mathbf{N}$, при $n, N \rightarrow \infty$ таких, что

$$
\sqrt{N}\left(\alpha_{n N}-a\right) \rightarrow C
$$

верно, что

$$
\begin{array}{ll}
\mathbf{P}\left\{\eta_{(n N)} \leqslant r\right\}=\left(1-P_{r}\right)^{N}\left(\left(\frac{1}{\Phi(C / \sigma)}\right)+o(1)\right), & a_{(\leqslant r)}<a \\
\mathbf{P}\left\{\eta_{(n N)} \leqslant r\right\}=\left(1-P_{r}\right)^{N}\left(\left(\frac{\Phi(C / \sigma(\leqslant r)}{\Phi(C / \sigma)}\right)+o(1)\right), & a_{(\leqslant r)}=a ; \\
\mathbf{P}\left\{\eta_{(N)} \leqslant r\right\}=\left(1-P_{r}\right)^{N} o(1), & a_{(\leqslant r)}>a .
\end{array}
$$

Пусть

$$
\begin{aligned}
L_{(\leqslant r)}(\lambda) & =e^{a_{(\leqslant r)} \lambda} \mathbf{E} e^{-\lambda \xi^{(\leqslant r)}}, & m_{(\leqslant r)}(\lambda) & =\left(\ln \left(L_{(\leqslant r)}(\lambda)\right)\right)^{\prime}, \\
\sigma_{(\leqslant r)}^{2}(\lambda) & =m_{(\leqslant r)}^{\prime}(\lambda), & \lambda & \geqslant 0 .
\end{aligned}
$$

Пусть $r \geqslant 1$,

$$
\mathbf{P}\left(\xi_{0}=0\right)>0, \quad \mathbf{P}\left(\xi_{0} \leqslant r\right)>\mathbf{P}\left(\xi_{0}=0\right) .
$$

Тогда $m_{\leqslant r}(\lambda)-$ возрастающая непрерывная функция, такая, что

$$
m_{(\leqslant r)}(0)=0, \quad \lim _{\lambda \rightarrow \infty} m_{(\leqslant r)}(\lambda)=a_{(\leqslant r)} .
$$

Пусть

$$
0<\alpha_{n N}<\min \left(a, a_{\leqslant r}\right)
$$

Обозначим

$$
\begin{aligned}
h & =m^{-1}\left(a-\alpha_{n N}\right), \\
h_{(\leqslant r)} & =m_{(\leqslant r)}^{-1}\left(a_{(\leqslant r)}-\alpha_{n N}\right), \\
\beta_{(\leqslant r)}\left(\alpha_{n N}\right) & =\frac{\left(1-e^{-h}\right)}{\left(1-e^{\left.-h_{(\leqslant r)}\right)} .\right.}
\end{aligned}
$$

Теорема 4. Предположим, что $r \geqslant 1$,

$$
\mathbf{P}\left(\xi_{0}=0\right)>0, \quad \mathbf{P}\left(\xi_{0} \leqslant r\right)>\mathbf{P}\left(\xi_{0}=0\right) .
$$

$\Pi y c m b$

$$
0<d<d_{1}<\min \left(a, a_{\leqslant r}\right) .
$$

Тогда для всех $r \in \mathbf{N}$, при $n, N \rightarrow \infty$

$$
\mathbf{P}\left\{\eta_{(n N)} \leqslant r\right\}=\mathbf{P}\left\{\eta_{(N)}^{\prime} \leqslant r\right\} \beta_{(\leqslant r)}\left(\alpha_{n N}\right)(1+o(1))
$$

равномерно по $d<\alpha_{n N}<d_{1}$. 
Теорема 5. Предположим, что $\xi=\xi(\theta)$ имеет распределение (6) и условие $A_{1}$ выполнено. Пусть $r \in \mathbf{N}$. Пусть $\theta=\theta(N)$ выьбрань так, что

$$
N p_{r+1}(\theta) \rightarrow \lambda, \quad 0<\lambda<\infty
$$

Тогда, если $n, N \rightarrow \infty$ так, что

$$
\frac{n}{N^{r /(r+1)}} \rightarrow \infty
$$

mo

$$
\mathbf{P}\left\{\eta_{(n N)}=r\right\}=e^{-\lambda}+o(1)
$$

$u$

$$
\mathbf{P}\left\{\eta_{(n N)}=r+1\right\}=1-e^{-\lambda}+o(1) .
$$

Теорема 6. Предположим, что $\xi=\xi(\theta)$ имеет распределение (6) и условие $A_{1}$ выполнено. Пусть $n \in \mathbf{N}$ фиксировано. Пусть $\theta=\theta(N)$ вылбрань так, что

$$
N p_{1}(\theta) \rightarrow \lambda, \quad 0<\lambda<\infty
$$

Тогда при $N \rightarrow \infty$

$$
\mathbf{P}\left\{\eta_{(n N)}=0\right\}=\frac{e^{-\lambda}}{\sum_{l=0}^{n} \frac{\lambda^{l} e^{-\lambda}}{l !}}+o(1)
$$

$u$

$$
\mathbf{P}\left\{\eta_{(n N)}=1\right\}=1-\frac{e^{-\lambda}}{\sum_{l=0}^{n} \frac{\lambda^{l} e^{-\lambda}}{l !}}+o(1) .
$$

В случае $r=0$ теорема 5 верна при более слабых условиях.

Теорема 7. Предположим, что $\xi=\xi(\theta)$ имеет распределение (6) и условие $A_{1}$ выполнено. Пусть $\theta=\theta(N)$ выбраны так, что

$$
N p_{1}(\theta) \rightarrow \lambda, \quad 0<\lambda<\infty
$$

Тогда при $n, N \rightarrow \infty$

$$
\mathbf{P}\left\{\eta_{(n N)}=0\right\}=e^{-\lambda}+o(1)
$$

$u$

$$
\mathbf{P}\left\{\eta_{(n N)}=1\right\}=1-e^{-\lambda}+o(1) .
$$




\section{3. Доказательства}

Доказательство теоремы 2. Используя формулу (15) из [4] для $\xi_{i}$ и для $\xi_{i}^{(\leqslant r)}$ в (5), получаем (7).

По закону больших чисел Колмогорова,

$$
\mathbf{P}\left\{S_{N}^{(\leqslant r)} \leqslant n\right\} \rightarrow 0
$$

если $n, N \rightarrow \infty$ так, что $d<\alpha_{n N}<d_{1}$. Используя этот факт и формулу (15) из [4] в (5), получаем (8).

Для доказательства теоремы 3 достаточно применить формулу (16) из [4] к $\xi_{i}$ и к $\xi_{i}^{(\leqslant r)}$ в (5).

Для доказательства теоремы 4 достаточно применить формулу (38) из [4] к $\xi_{i}$ и $\xi_{i}^{(\leqslant r)}$ в (5) с учетом (4).

Доказательство теоремы 5. Заметим, что

$$
B(\theta)=b_{0}+o(1), \quad \theta=\left(\frac{(r+1) !\left(b_{0} \lambda+o(1)\right)}{N b_{r+1}}\right)^{1 /(r+1)}
$$

Подставляя $r+1$ вместо $r$ в формулу (52) в [4], получаем

$$
\mathbf{P}\left\{S_{N}>n\right\} \leqslant \frac{1}{n} \mathbf{E} S_{N}=o(1) .
$$

Пусть $r_{1} \in \mathbf{N}$. Тогда верны соотношения

$$
\begin{aligned}
\mathbf{P}\left\{S_{N}^{\left(\leqslant r_{1}\right)}>n\right\} & \leqslant \frac{N}{n} \mathbf{E} \xi^{\left(\leqslant r_{1}\right)} \\
& =\frac{N}{n} \frac{\sum_{k=1}^{r_{1}} k \frac{b_{k}}{k ! b_{0}}\left(\left(\frac{(r+1) !\left(b_{0} \lambda+o(1)\right)}{N b_{r+1}}\right)^{1 /(r+1)}\right)^{k}}{\sum_{k=0}^{r_{1}} \frac{b_{k}}{k ! b_{0}}\left(\left(\frac{(r+1) !\left(b_{0} \lambda+o(1)\right)}{N b_{r+1}}\right)^{1 /(r+1)}\right)^{k}}(1+o(1))=o(1)
\end{aligned}
$$

и

$$
\begin{aligned}
\left(1-P_{r-1}\right)^{N} & =o(1) \\
\left(1-P_{r}\right)^{N} & =e^{-\lambda}+o(1), \\
\left(1-P_{r+1}\right)^{N} & =1+o(1) .
\end{aligned}
$$

Тогда, в силу (9) и (10),

$$
\begin{aligned}
\mathbf{P}\left\{S_{N} \leqslant n\right\} & =1+o(1), \\
\mathbf{P}\left\{S_{N}^{\left(\leqslant r_{1}\right)} \leqslant n\right\} & =1+o(1),
\end{aligned}
$$


Применив эти оценки и (11) к (5), получаем

$$
\begin{aligned}
\mathbf{P}\left\{\eta_{(n N)} \leqslant r-1\right\} & =o(1), \\
\mathbf{P}\left\{\eta_{n(N)} \leqslant r\right\} & =e^{-\lambda}+o(1), \\
\mathbf{P}\left\{\eta_{(n N)} \leqslant r+1\right\} & =1+o(1) .
\end{aligned}
$$

Последние оценки доказывают теорему 5.

Доказательство теоремьл 6. Справедливы соотношения

$$
\begin{aligned}
\left(1-P_{-1}\right)^{N} & =0 \\
\left(1-P_{0}\right)^{N} & =e^{-\lambda}+o(1) \\
\left(1-P_{1}\right)^{N} & =1+o(1)
\end{aligned}
$$

и

$$
\mathbf{P}\left\{\eta_{(n N)} \leqslant-1\right\}=0 .
$$

Применив пуассоновскую предельную теорему и (12) к (5), получаем

$$
\mathbf{P}\left\{\eta_{(n N)} \leqslant 0\right\}=\frac{e^{-\lambda}}{\sum_{l=0}^{n} \frac{\lambda^{l} e^{-\lambda}}{l !}}+o(1) .
$$

Заметим, что $\xi_{1}^{(\leqslant 1)}, \ldots, \xi_{N}^{(\leqslant 1)}-$ независимые индикаторы, такие, что

$$
N \mathbf{P}\left\{\xi_{i}^{(\leqslant 1)}=1\right\} \rightarrow \lambda
$$

при $N \rightarrow \infty$. Следовательно, по пуассоновской предельной теореме

$$
\mathbf{P}\left\{S_{N}^{(\leqslant 1)} \leqslant n\right\}=\sum_{l=0}^{n} \frac{\lambda^{l} e^{-\lambda}}{l !}+o(1) .
$$

Применив (12), (15) и пуассоновскую предельную теорему к (5), получаем

$$
\mathbf{P}\left\{\eta_{(n N)} \leqslant 1\right\}=(1+o(1)) \frac{\sum_{l=0}^{n} \frac{\lambda^{l} e^{-\lambda}}{l !}+o(1)}{\sum_{l=0}^{n} \frac{\lambda^{l} e^{-\lambda}}{l !}+o(1)}=1+o(1) .
$$

Из (13), (14) and (16) следует теорема 6.

Доказательство теоремы 7 повторяет доказательство теоремы 6. 


\section{Список литературы}

1. Колчин В. Ф., Один класс предельных теорем для условных распределений. Литовский матем. сборник (1968) 8, №1, 53-63.

2. Колчин В. Ф., Случайные графы. Физматлит, Москва, 2000.

3. Тимашев А. Н., Асимптотические разложения в вероятностной комбинаторике. ТВП, Москва, 2011.

4. Чупрунов А. Н., Фазекаш И., Аналог обобщенной схемы размещения. Предельные теоремы для числа ячеек заданного объема. Дискретная математика (2012) 24, №1, 140-158.

5. Колчин А. В., Предельные теоремы для обобщенной схемы размещения. Дискретная математика (2003) 15, №4, 143-157.

6. Колчин А. В., Колчин В. Ф., О переходе распределений сумм независимых одинаково распределенных случайных величин с одной решетки на другую в обобщенной схеме размещения. Дискретная математика (2006) 18, №4, 113-127.

7. Колчин А. В., Колчин В. Ф., Переход с одной решетки на другую распределений сумм случайных величин, встречающихся в обобщенной схеме размещения. Дискретная математика (2007) 19, №3, 15-21.

8. Chuprunov A. N., Fazekas I., An inequality for moments and its applications to the generalized allocation scheme. Publ. Math. Debrecen (2010) 76, №3, 271-286.

9. Erdős P., Rényi A., On the evolution of random graphs. Publ. Math. Inst. Hung. Acad. Sci. (1960) 5, 17-61.

Статья поступила 18.05.2011. 